\title{
Mystery Case: A 48-year-old woman with bizarre behavior, neurologic symptoms, and progressive decline
}

Navin K. Kesari, MD,* Tiffany Fleet, PhD,* Bader Alenzi, MD, and J. Clay Goodman, MD

Neurology ${ }^{\circledR}$ 2018;90:242-247. doi:10.1212/WNL.0000000000004886

\section{Section 1}

A 48-year-old woman with an alcohol use disorder presented with a subacute onset of altered mental status. Two months prior to presentation, the patient recreationally used morphine, oxycodone, and heroin over the span of 1 week. At the end of this 7-day period, she experienced an episode of lethargy for approximately 24 hours and continued to be difficult to arouse during the following 2 days; however, she did not seek medical attention. She returned to her baseline functional status and remained asymptomatic until 3 weeks later, when she fell and hit her head, initially resulting in a headache. One day later, she began exhibiting bizarre behavior that gradually increased in severity over the following month. She began by sleeping with a recently deceased relative's ashes and emotional labiality and progressed to brushing her teeth with a comb, showering while dressed, undressing and then going outside, and not recognizing family members. Her husband brought her to the hospital for further evaluation.

Her neurologic examination demonstrated that she was only oriented to self and had poor comprehension due to an inability to follow commands or respond appropriately to questions. Moreover, she demonstrated apraxia, aphasia, and selective mutism. Additional neurologic findings included patellar hyperreflexia, a palmomental reflex, and a wide-based gait. The remainder of the examination was limited by patient participation.

\section{Questions for consideration:}

1. What is your initial differential diagnosis?

2. What laboratory studies would you perform to reach a final diagnosis?

\author{
Correspondence \\ Dr. Kesari \\ navin.kesari@bcm.edu
}

\section{MORE ONLINE}

Survey and results

NPub.org/MC9005 


\section{Section 2}

The initial differential diagnosis included infectious, malignant, metabolic, demyelinating, and psychiatric etiologies. The patient's urine drug screen was negative for toxins and a urinalysis showed signs of a urinary tract infection (positive nitrates, 2+ leukocytes, 68 white blood cells/high-power field, and many bacteria). CSF analysis was inconsistent with infectious, malignant, or inflammatory etiologies (table e-1, links.lww.com/WNL/A94). Serum infectious, serologic, and autoimmune markers were inconclusive for a definitive etiology (table e-1). There were no detectable signs of poisoning in the form of carboxyhemoglobin or heavy metals including lead, arsenic, and mercury (table e-1).

We performed a series of electrophysiologic and imaging studies to further characterize the patient's neurologic symptoms since biochemical analysis of blood, urine, and CSF were unrevealing. An EEG demonstrated intermittent, generalized, reactive polymorphic delta slowing suggestive of encephalopathy. Brain MRI without contrast showed confluent supratentorial white matter signal changes with mild diffuse cortical swelling indicative of diffuse encephalitis (figure, A-C). The MRI was repeated 1 week later and revealed unchanged nonenhancing confluent supratentorial white matter T2 hyperintensities with restricted diffusion and sparing of the U-fibers. There was no evidence of enhancing lesions on the spine MRI or vasculitis seen on the cerebral angiogram.

\section{Questions for consideration:}

1. What is your differential diagnosis after these studies?

2. Are there any other diagnostic studies that would provide a definitive diagnosis?

Figure MRI and hematoxylin \& eosin (H\&E) of a patient with neurocognitive decline, apraxia, aphasia, and hyperreflexia
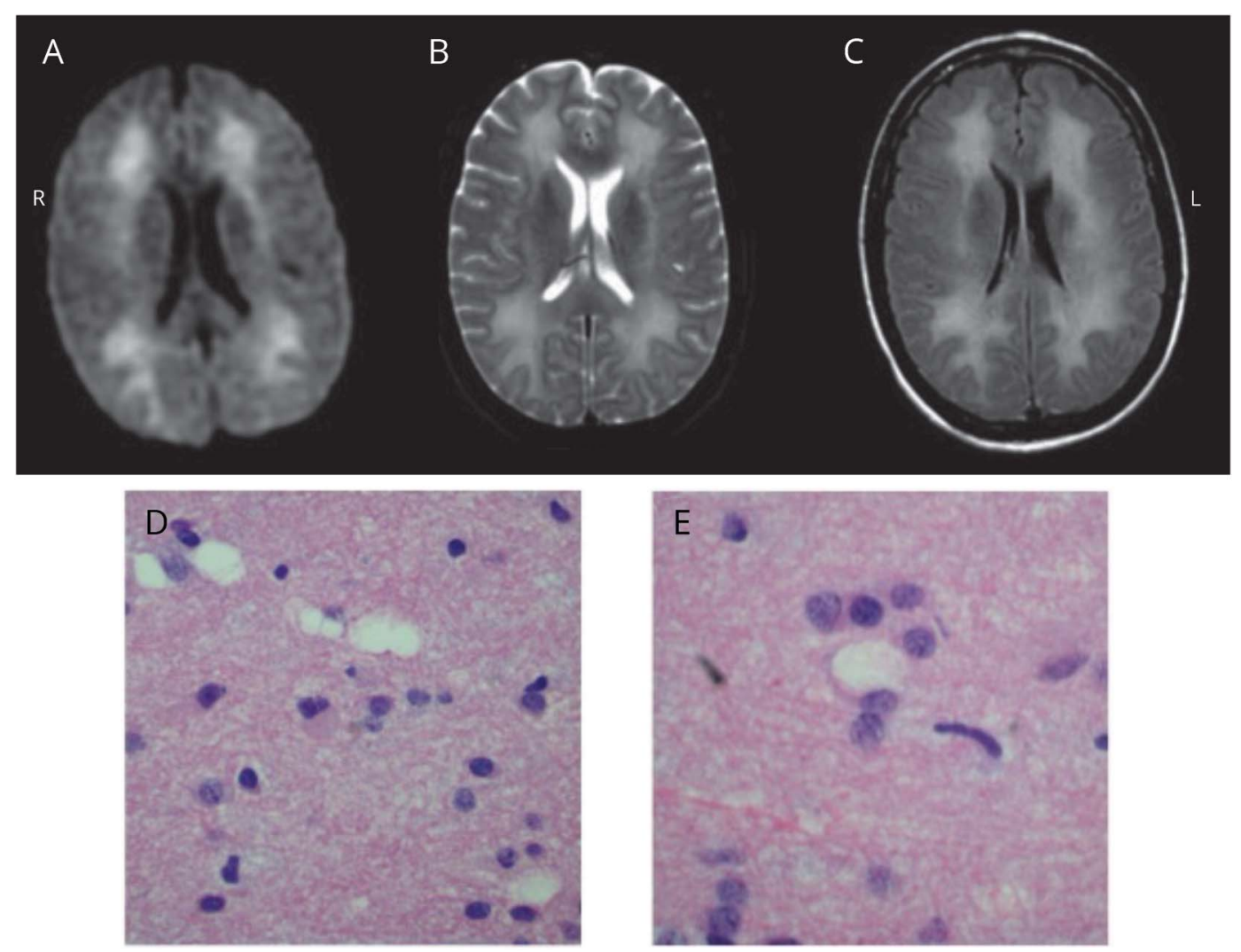

Axial MRI with diffusion-weighted imaging (A), T2 (B), and T2 fluid-attenuated inversion recovery (C) sequences. Each series shows bilateral, symmetric periventricular white matter hyperintensities. H\&E-stained sections at $\times 200$ (D) and $\times 400$ (E) show hypercellular white matter due to astrogliosis and microglial activation.

GO TO SECTION 3 


\section{Section 3}

The identification of leukoencephalopathy on brain MRI considerably narrowed the differential diagnosis, but many neurologic conditions are identified by similar radiologic findings. These include demyelinating, inflammatory, hypoxic, metabolic, genetic, and substance-induced conditions. ${ }^{1}$ Pathologies such as multiple sclerosis, progressive multifocal leukoencephalopathy (PML), posterior reversible encephalopathy syndrome (PRES), primary angiitis of the CNS (PACNS), and cerebral autosomal dominant arteriopathy with subcortical infarcts and leukoencephalopathy (CADASIL) were in the differential diagnosis. In addition, we considered other inherited etiologies such as vanishing white matter disease and adult-onset autosomal dominant leukodystrophy (ADLD).
With the broad differential diagnosis and limited clinical and radiologic distinguishing features, we performed a brain biopsy. Pathologic analysis of the brain biopsy demonstrated numerous white matter vacuoles occurring singly and in groups, and more white matter astrogliosis and microglial activation compared to the cortex (figure). There were no lymphocytes, giant cells, macrophages, viral inclusions, or hypoxic or ischemic changes present. Luxol fast blue staining showed patchy myelin loss (not shown).

\section{Question for consideration:}

1. What is your final diagnosis and treatment?

GO TO SECTION 4 


\section{Section 4}

The patient presented with a 1-month history of altered mentation and nonfocal neurologic symptoms almost 2 months after a suspected opioid overdose and a head injury. She was treated empirically with IV corticosteroids, but 1 week after admission, the patient exhibited autonomic instability, diffuse hyperreflexia, decorticate posturing, tremulousness, and stuporous mentation. She was transferred to the intensive care unit, intubated, and treated with lorazepam and other supportive care measures. The patient did not recover and died 7 weeks after her initial presentation. The history, MRI findings, brain biopsy pathology, and clinical progression indicated that the diagnosis of spongiform leukoencephalopathy was likely a result of opioid use.

\section{Discussion}

In a 46-year-old woman with no chronic medical conditions, the subacute, rapid neurologic decline presented a diagnostic dilemma. Her symptoms were not suggestive of a demyelinating disease such as multiple sclerosis because she did not have ophthalmologic involvement, relapsing and remitting symptoms, gradually progressing neurologic deficits, or demyelination on MRI. She had no history of being immunocompromised due to HIV, use of immunosuppressive therapy, or malignancy that could predispose her to JC virus-induced PML. The patient's symptoms and clinical course made PRES an unlikely diagnosis since she did not have hypertension, cerebral ischemia, or use of immunosuppressive therapy. Moreover, she did not have the predominant posterior cerebral white matter edema characteristic of PRES.

Vasculitides such as PACNS and secondary vasculitis due to inflammatory or infectious etiology were excluded with a 4-vessel cerebral angiography. CADASIL typically presents with migraines, imaging showing multiple infarcts over time, and a family history of strokes, but the patient did not have these clinical characteristics. Inherited disorders such as vanishing white matter disease; ADLD; fragile $\mathrm{X}$-associated tremor/ataxia syndrome (X-linked); mitochondrial myopathy; encephalopathy, lactic acidosis, and stroke-like episodes; metachromatic leukodystrophy; Krabbe disease; and Fabry disease were precluded by age at onset and clinical and radiologic characteristics further described in Nannucci et al. ${ }^{1}$ In addition, an etiology such as poisoning with carbon monoxide or heavy metals were excluded by history and serum studies. After an extensive series of diagnostic studies, a brain biopsy and history of opioid use provided the final diagnosis of toxic leukoencephalopathy.

Toxic leukoencephalopathy (TLE) represents a group of CNS white matter disorders with varying presentations. Substances inducing TLE include, but are not limited to, benzodiazepines, methadone, oxycodone, carbon monoxide, and methotrexate. Currently, there is no unifying mechanism that explains how a variety of drugs and toxins result in TLE, and moreover, it is difficult to predict which patients will develop TLE, as many people are exposed to these agents, but relatively few cases have been reported. Our patient demonstrates specific clinical, radiologic, and pathologic characteristics similar to a TLE due to heroin use.

There are 3 stages in the clinical pathogenesis of heroininduced TLE, ${ }^{2}$ which were all exhibited by our patient. The first stage initiates with cerebellar dysfunction, including limb ataxia, gait disturbances, dysdiadochokinesia, and soft pseudobulbar speech. The second stage shows worsening cerebellar syndrome, hyperreflexia, primitive reflexes, choreoathetosis, myoclonic jerks, and spastic paresis. Akinetic mutism, hyperpyrexia, and decorticate posturing characterize the final stage, which is usually terminal. ${ }^{2,3}$ MRI typically shows nonenhancing T2 hyperintensities in the posterior cerebral hemispheres and cerebellum, which correspond to the early cerebellar symptoms observed in these patients. In addition, sparing of the U-fibers and other gray matter areas is typically associated with hypoxic insult. ${ }^{3,4}$ Brain histologic changes include vacuolization, astrogliosis, decreased oligodendrocytes and axons, and an absence of myelin breakdown products. ${ }^{5,6}$ There is no validated treatment for heroin-induced TLE and it has an estimated mortality rate of $23 \%{ }^{2}$

Although the exact extent and method of opioid ingestion for this patient is unknown, there were multiple reports of morphine, oxycodone, and heroin use, which likely resulted in TLE in the patient. This presentation differs from other TLE reports because the patient experienced a delayed onset of symptoms, which were precipitated by a mild head injury. This is not an isolated phenomenon since a delayed spongiform leukoencephalopathy has been described in an accidental methadone overdose in a child and in an overdose in an IV heroin drug user. ${ }^{7,8}$ The radiologic findings differ from some cases of heroin-induced TLE since the hypointensities found on T2 fluid-attenuated inversion recovery were supratentorial and there was no cerebellar involvement; however, there is a case report of methadone-induced TLE resulting in diffuse $\mathrm{T} 2$ hyperintensities with sparing of cortical U-fibers. ' Moreover, periventricular white mater changes have been documented by Kriegstein et al. ${ }^{3}$ Even though there is some uncertainty about the method of heroin ingestion and differences in the radiologic findings of heroin leukoencephalopathy, the patient history and physical indicate that opioid use resulted in a delayed onset of TLE.

With the recent growth in the opioid epidemic, there is an increased urgency for the recognition of the devastating consequences of both prescription opioid and heroin misuse. Recently, the Surgeon General published a call to action to end the opioid crisis plaguing the nation in The New England Journal of Medicine. According to Surgeon General Murthy's report, the number of opioid overdoses has increased 4 times over since 2000, 2 million people are addicted to prescription opioids, and the prescription opioid crisis is contributing to 
the resurgence in heroin addiction. ${ }^{10}$ Moreover, public and physician awareness of this relatively unknown consequence of opioid use may help identify more patients at risk for TLE.

\section{Author contributions}

Navin Kesari and Bader Alenzi wrote section 1 and formatted the table and imaging figure. Navin Kesari and Tiffany Fleet wrote sections $2-4$ and provided manuscript revision. Tiffany Fleet wrote the discussion section and formatted the table. Clay Goodman provided the pathology images and manuscript revision.

\section{Study funding}

This research was supported by a grant from the NIH (F31DK107254 to T.F.). Partial support for this work was provided by a grant to Baylor College of Medicine from the Medical Scientist Training Program NIGMS T32 GM7330.

\section{Disclosure}

The authors report no disclosures relevant to the manuscript. Go to Neurology.org/N for full disclosures.

\section{References}

1. Nannucci S, Donnini I, Pantoni L. Inherited leukoencephalopathies with clinical onset in middle and old age. J Neurol Sci 2014;347:1-13.

2. Wolters EC, van Wijngaarden GK, Stam FC, et al. Leucoencephalopathy after inhaling "heroin" pyrolysate. Lancet 1982;2:1233-1237.

3. Kriegstein AR, Shungu DC, Millar WS, et al. Leukoencephalopathy and raised brain lactate from heroin vapor inhalation ("chasing the dragon"). Neurology 1999;53: 1765-1773.

4. Kriegstein AR, Armitage BA, Kim PY. Heroin inhalation and progressive spongiform leukoencephalopathy. N Engl J Med 1997;336:589-590.

5. Buttner A, Mall G, Penning R, Weis S. The neuropathology of heroin abuse. Forensic Sci Int 2000;113:435-442.

6. Halloran O, Ifthikharuddin S, Samkoff L. Leukoencephalopathy from "chasing the dragon." Neurology 2005;64:1755.

7. Zanin A, Masiero S, Severino MS, Calderone M, Da Dalt L, Laverda AM. A delayed methadone encephalopathy: clinical and neuroradiological findings. J Child Neurol 2010;25:748-751.

8. Barnett MH, Miller LA, Reddel SW, Davies L. Reversible delayed leukoencephalopathy following intravenous heroin overdose. J Clin Neurosci 2001;8:165-167.

9. Metkees M, Meesa IR, Srinivasan A. Methadone-induced acute toxic leukoencephalopathy. Pediatr Neurol 2015;52:256-257.

10. Murthy VH. Ending the opioid epidemic: a call to action. N Engl J Med 2016;375: 2413-2415.

\section{Mystery Case responses}

The Mystery Case series was initiated by the Neurology ${ }^{\circledR}$ Resident \& Fellow Section to develop the clinical reasoning skills of trainees. Residency programs, medical student preceptors, and individuals were invited to use this Mystery Case as an educational tool. Responses to multiple choice questions formulated using this case were solicited through a group e-mail sent to the American Academy of Neurology Consortium of Neurology Residents and Fellows and through social media. We received 58 responses. Most of the respondents ( $83 \%$ ) had been in practice for $1-4$ years; $78 \%$ were residents or fellows while $17 \%$ were faculty/boardcertified physicians. Fifty percent resided outside the United States. A wide range of practice settings was represented.

When presented with information about this patient's history and physical examination (Section 1) and asked what top 4 investigations they would want to order aside from standard bloodwork, the most popular choices were MRI brain (91\%), urine toxicology screen (81\%), lumbar puncture (78\%), and EEG (74\%). These represented an ideal combination of investigations for a patient presenting with a history of substance abuse presenting with altered cognition/behavior and associated CNS signs. Twenty-nine percent opted for CT head, a less sensitive but more readily available imaging modality than MRI.

Upon being shown the results of the patient's blood/urine tests, EEG (intermittent generalized reactive polymorphic delta slowing), brain MRI (figure, A-C), and 4-vessel cerebral angiography (reported normal), respondents were then asked about their top 4 differential diagnoses. The top choices were progressive toxic leukoencephalopathy (79\%), PML (52\%), ADLD (45\%), PRES (29\%), PACNS (29\%), Wernicke encephalopathy (28\%), metachromatic leukodystrophy (24\%), and cerebral autosomal dominant arteriopathy with subcortical infarcts and leukoencephalopathy (24\%). Indeed, toxic leukoencephalopathy would be the primary consideration in this woman with opioid abuse. Diagnoses like PML and PRES are reasonable considerations given the combination of subacute encephalopathy and symmetric white matter changes. Wernicke encephalopathy is a key treatable diagnosis to consider, but the neuroimaging findings are less florid, typically involving the mammillary bodies, medial thalami, region around the third and fourth ventricles, and periaqueductal gray matter. ${ }^{1}$ Whereas PACNS is an important consideration, the fact that 4-vessel cerebral angiography was normal makes this unlikely. CADASIL can also present with white matter changes and cognitive deterioration though there was no history of recurrent strokes or migraines. $\mathrm{ADLD}$ is a less appropriate consideration here, given that it is generally slowly progressive and has relatively mild cognitive impairment in addition to associated autonomic dysfunction or ataxia. ${ }^{2}$

On then being asked what one additional test they would order at this point to settle the diagnosis in this complicated case, $31 \%$ of respondents appropriately chose brain biopsy. Other choices included magnetic resonance spectroscopy (17\%, expensive while potentially inconclusive), repeat CSF and serum autoantibody testing (17\%, likely diminishing returns at this point), and CSF 14-3-3 testing (14\%, inappropriate as imaging/EEG is inconsistent with prion disease). ${ }^{3}$ Finally, upon being presented the brain biopsy results (figure, D-E) — which just show astrogliosis and microglial activation - and asked what the final diagnosis would be, the top choice appropriately remained toxic leukoencephalopathy (40\%). The next most popular choice was PML (17\%); however, this typically has the histopathologic triad of demyelination, bizarre astrocytes, and enlarged oligodendroglial nuclei, with JC virus being demonstrated by immunohistochemistry and in situ hybridization. ${ }^{4}$

This case highlights both the potential delayed presentation of toxic leukoencephalopathy following drug 
abuse and the diagnostic challenges posed by competing differential diagnoses for altered mental status with white matter changes.

Aravind Ganesh, MD

Department of Clinical Neurosciences, University of Calgary, Canada; Centre for Prevention of Stroke and Dementia, Nuffield Department of Clinical Neurosciences, University of Oxford, UK

\section{References}

1. Luigetti M, De Paulis S, Spinelli P, et al. Teaching neuroimages: the full-blown neuroimaging of Wernicke encephalopathy. Neurology 2009;72:e115.

2. Lin ST, Ptacek LJ, Fu YH. Adult-onset autosomal dominant leukodystrophy: linking nuclear envelope to myelin. J Neurosci 2011;31:1163-1166.

3. Vitali P, Maccagnano E, Caverzasi E, et al. Diffusion-weighted MRI hyperintensity patterns differentiate CJD from other rapid dementias. Neurology 2011;76: $1711-1719$.

4. Berger JR, Aksamit AJ, Clifford DB, et al. PML diagnostic criteria: consensus statement from the AAN neuroinfectious disease section. Neurology 2013;80:1430-1438.

\section{Disputes \& Debates: Rapid online correspondence}

The editors encourage comments on recent articles through Disputes \& Debates:

Access an article at Neurology.org/N and click on "COMMENT" beneath the article header. Responses will be posted within 3 business days.

Before submitting a comment to Disputes \& Debates, remember the following:

- Disputes \& Debates is restricted to comments about studies published in Neurology within the last eight weeks

- Read previously posted comments; redundant comments will not be posted

- Your submission must be 200 words or less and have a maximum of five references; reference one must be the article on which you are commenting

- You can include a maximum of five authors (including yourself)

\section{Subspecialty Alerts by E-mail!}

Customize your online journal experience by signing up for e-mail alerts related to your subspecialty or area of interest. Access this free service by clicking on the "My Alerts" link on the home page. An extensive list of subspecialties, methods, and study design choices will be available for you to choose from-allowing you priority alerts to cutting-edge research in your field!

\section{Did You Know...}

... you can browse by subspecialty topics on Neurology.org?

Go to: Neurology.org and click on "Topics" in the top navigation bar. 


\section{Neurology}

\section{Mystery Case: A 48-year-old woman with bizarre behavior, neurologic symptoms, and progressive decline \\ Navin K. Kesari, Tiffany Fleet, Bader Alenzi, et al. Neurology 2018;90;242-247 \\ DOI 10.1212/WNL.0000000000004886}

This information is current as of January 29, 2018

Updated Information \& Services

References

Subspecialty Collections

Permissions \& Licensing

Reprints including high resolution figures, can be found at: http://n.neurology.org/content/90/5/242.full

This article cites 14 articles, 5 of which you can access for free at: http://n.neurology.org/content/90/5/242.full\#ref-list-1

This article, along with others on similar topics, appears in the following collection(s):

All Clinical Neurology

http://n.neurology.org/cgi/collection/all_clinical_neurology All Neuropsychology/Behavior

http://n.neurology.org/cgi/collection/all_neuropsychology_behavior MRI

http://n.neurology.org/cgi/collection/mri

Opiates

http://n.neurology.org/cgi/collection/opiates

Information about reproducing this article in parts (figures,tables) or in its entirety can be found online at:

http://www.neurology.org/about/about_the_journal\#permissions

Information about ordering reprints can be found online:

http://n.neurology.org/subscribers/advertise

Neurology ${ }^{\circledR}$ is the official journal of the American Academy of Neurology. Published continuously since 1951, it is now a weekly with 48 issues per year. Copyright (O 2018 American Academy of Neurology. All rights reserved. Print ISSN: 0028-3878. Online ISSN: 1526-632X.

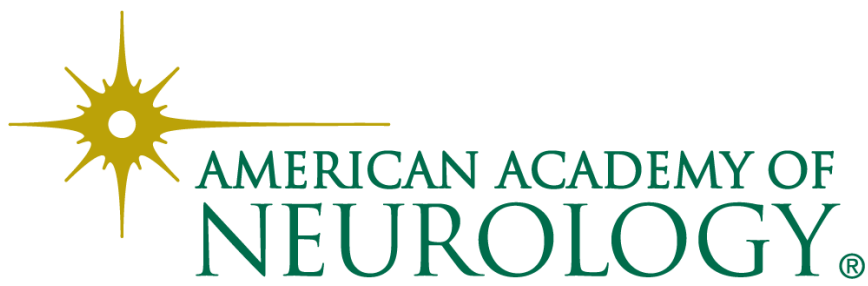

